\title{
EFICIÊNCIA HOSPITALAR NO SUS: ANÁLISE DE 10 HOSPITAIS DO MIX PÚBLICO-PRIVADO DO ESTADO DE MATO GROSSO
}

\author{
HOSPITAL EFFICIENCY IN THE SUS: AN ANALYSIS OF 10 HOSPITALS OF THE PRIVATE-PUBLIC MIX IN THE STATE OF \\ MATO GROSSO
}

\section{EFICACIA DEL HOSPITAL EN EL SUS: ANÁLISIS DE 10 HOSPITALES DEL MIX PÚBLICO-PRIVADO EN MATO GROSSO}

\section{RESUMO}

A assistência hospitalar do Sistema Único de Saúde (SUS) tem diante de si um desafio: aumento da eficiência. Para verificar a situação dos hospitais que compõem o mix público-privado do SUS no estado de Mato Grosso, comparando a eficiência de 10 hospitais distribuídos entre públicos, privados e filantrópicos, este trabalho aplicou a Análise Envoltória de Dados, do inglês Data Envelopment Analysis (DEA), metodologia que pode incorporar múltiplas entradas e saídas. A medida de eficiência por esse método considera 0 mix de entradas e saídas, sendo mais abrangente e confiável do que um conjunto de taxas operacionais ou medidores de lucratividade, e aplicável a diversos tipos de organizações públicas e sem fins lucrativos. Este trabalho caracteriza-se como um estudo multicaso descritivo e exploratório com abordagem quantitativa. Foram selecionados 10 hospitais distribuídos entre públicos, privados e filantrópicos, localizados em três regiões de saúde do estado de Mato Grosso, estratégia que permitiu comparar a eficiência observada entre as três categorias de hospitais. No ano de 2011, os hospitais filantrópicos (1) e privados $(0,96)$ apresentaram scores de eficiência melhores que os públicos $(0,84)$. 0 mesmo ocorreu no primeiro semestre de 2012, quando os privados $(0,95)$ e filantrópicos $(0,92)$ inverteram sua posição, mas continuaram com melhor desempenho que os públicos $(0,81)$. Em ambos os períodos, os públicos apresentaram o menor nível de eficiência total. Assim, nesse modelo de análise DEA aplicado no conjunto dos hospitais pesquisados, tanto os hospitais privados quanto os filantrópicos apresentaram um patamar de eficiência mais elevado do que os hospitais públicos, 0 que é discutido.

PALAVRAS-CHAVE: Sistema Único de Saúde, assistência hospitalar, eficiência organizacional, administração hospitalar, Análise Envoltória de Dados.

Paulo Cesar Souza paulobbg@unemat.br

Doutor em Ciências da Saúde pela Universidade Federal de Mato Grosso - Cuiabá - MT, Brasil

João Henrique Gurtler Scatena jscatena@terra.com.br

Professor da Universidade Federal de Mato Grosso, Departamento de Saúde Coletiva - Cuiabá - MT, Brasil

Ruth Terezinha Kehrig ruth.kehrig@gmail.com

Professora da Universidade Federal de Mato Grosso, Instituto de Saúde Coletiva - Cuiabá - MT, Brasil

Submetido 16.02.2017. Aprovado 24.07.2017

Avaliado pelo processo de double blind review.

DOI: http://dx.doi.org/10.12660/cgpc.v22n72.66242 


\section{Abstract}

The hospital care provided by Brazil's Unified Health System (SUS) faces a challenge: to increase efficiency. In order to examine the situation of hospitals forming SUS" public-private mix in the Brazilian state of Mato Grosso, this article compared the efficiency of 10 hospitals, including public, private and philanthropic institutions, using Data Envelopment Analysis (DEA), a method that works with multiple inputs and outputs. In this method, the efficiency rate considers the mix of inputs and outputs, being more comprehensive and reliable than a set of operating or profitability indices, and it is applicable to various types of public and nonprofit organizations. The present work is a descriptive and exploratory multicase study with a quantitative approach. We selected 10 public, private or philanthropic hospitals in three health regions in the state of Mato Grosso. This allowed comparing the efficiency found in the three hospital categories. In 2011, the philanthropic (1) and private hospitals (0.96) presented better efficiency scores than the public ones (0.84). The same occurred in the first half of 2012, when the private (0.95) and philanthropic (0.92) hospitals swapped positions but continued to perform better than the public ones (0.81). In both periods, the public hospitals had the lowest total efficiency. level. Thus, in this model of DEA analysis applied to all hospitals surveyed, the private and philanthropic hospitals had a higher efficiency level than the public hospitals, which we discuss in the article.

Keywords: Unified Health System, hospital care, organizational efficiency, hospital administration, Data Envelopment Analysis.

\section{Resumen}

La asistencia hospitalaria del Sistema Único de Salud (SUS) tiene ante síun desafío: el aumento de la eficiencia. Para verificar la situación de los hospitales que componen el mix público-privado del SUS en el estado de Mato Grosso, comparando la eficiencia de 10 hospitales distribuidos entre públicos, privados y filantrópicos, este trabajo aplicó el Análisis Envolvente de Datos, del inglés Data Envelopment Analysis (DEA), metodología que puede incorporar múltiples entradas y salidas. La medida de eficiencia por este método considera el mix de entradas y salidas, por lo que es más completo y confiable que un conjunto de tasas operativas o medidores de rentabilidad, y puede aplicarse a diversos tipos de organizaciones públicas y sin fines de lucro. Este trabajo se caracteriza como un estudio multicaso descriptivo y exploratorio con abordaje cuantitativo. Se seleccionaron 10 hospitales distribuidos entre públicos, privados y filantrópicos, ubicados en tres regiones de salud del estado de Mato Grosso, estrategia que permitio comparar la eficiencia observada entre las tres categorías de hospitales. En 2011, Ios hospitales de caridad (1) y, privados $(0,96)$ tuvieron puntuaciones de eficiencia mejores que los públicos (0,84). Lo mismo ocurrió en el primer semestre de 2012, cuando los privados $(0,95)$ y filantrópicos $(0,92)$ invirtieron su posición pero continuaron con mejor desempeño que los públicos $(0,81)$. En ambos períodos, los publicos presentaron el menor nivel de Eficiencia Total. As í, en ese modelo de análisis DEA aplicado al conjunto de los hospitales investigados, tanto los hospitales privados como los filantrópicos presentaron un nivel de eficiencia más elevado que los hospitales públicos, lo que se discute.

Palabras clave: Sistema Único de Salud, asistencia hospitalaria, eficiencia organizacional, administración hospitalaria, Análisis Envoltorio de Datos.

\section{INTRODUÇÃO}

Mesmo diante das muitas conquistas alcançadas pelo Sistema Único de Saúde (SUS), há um desafio ainda presente, principalmente no que se refere à assistência hospitalar: aumento da eficiência. Esse desafio foi evidenciado em publicação do Conselho Nacional de Secretários de Saúde (Conass, 2006) há quase uma década, mas ainda é uma realidade.

Diante disso, a avaliação em saúde mos- tra-se uma alternativa interessante a fim de conhecer melhor a realidade da assistência hospitalar quanto ao nível de eficiência observada.

Avaliar é "expor um valor assumido a partir do julgamento realizado com base em critérios previamente definidos" (Tanaka \& Melo, 2001, p. 12).

Embora pareça óbvio, avaliar é julgar, atribuir valor. Assim, para que determinado estudo seja considerado uma avaliação, é necessá- 
rio que seja realizado o julgamento, emitido juízo de valor. No entanto, para que seja possível julgar, necessita-se da elaboração de parâmetros que, fundados em teorias acerca da intervenção, sejam capazes de produzir indicadores válidos para o julgamento $(\mathrm{Ne}-$ mes, 2001).

Desse modo, a avaliação é "um processo por meio do qual se determina o valor de algo" (Malik \& Schiesari, 1998, p. 1). Segundo essa visão, a avaliação trata de determinar se um dado programa (ou ação, intervenção, atividade), descrito e especificado, ao qual se apõe uma definição prévia sobre como deveria ser, é bom, mau, correto, incorreto, vale ou não a pena, ou se está sendo realizado da maneira mais eficiente ou mais eficaz.

A despeito de toda a diversidade e de discordâncias sobre os vários aspectos da avaliação, para alguns autores, existe consenso com relação ao fato de que avaliar significa emitir um juízo de valor sobre uma intervenção ou um de seus componentes. Nota-se também que, para que sejam possíveis a realização da avaliação e a emissão de juízo de valor, torna-se necessário estabelecer padrões, objetivos, metas e construir indicadores que sirvam de base para o processo avaliativo (Furtado, 2001).

$\mathrm{Na}$ saúde, ao avaliar, utilizam-se teorias, conceitos e instrumentos de diversas outras áreas do conhecimento. Assim, a avaliação deve ser compreendida como uma área de aplicação, e não como uma ciência (Tanaka \& Melo, 2001).

Diante da necessidade da aplicação da avaliação, a fim de monitorar os níveis de efici- ência da assistência hospitalar do SUS, surgiu a questão norteadora deste trabalho: No grupo de 10 hospitais pesquisados, distribuídos entre públicos, privados e filantrópicos vinculados ao SUS, há diferença quanto aos níveis de eficiência?

A fim de responder a essa pergunta, este trabalho se propôs a aplicar na avaliação em saúde uma técnica de outra área de conhecimento, a fim de avaliar a eficiência hospitalar: a Análise Envoltória de Dados (DEA).

\section{ANÁLISE ENVOLTÓRIA DE DADOS}

A mensuração da eficiência constitui um dos grandes desafios no âmbito dos serviços de saúde. Uma das estratégias utilizadas é a mensuração da produtividade parcial, a qual, segundo Fitzsimmons e Fitzsimmons (2005), é similar à medida da eficiência de um sistema. Essa medida pode ser estabelecida como a razão das saídas pelas entradas, sendo expressa pelo quociente da produção pelo insumo empregado, ou seja, produção dividida pelo insumo. Na saúde, é bastante comum utilizar-se de indicadores dessa natureza, como: número de consultas por médico, procedimentos por hora trabaIhada, exames por dia etc. Nessa forma de cálculo, realiza-se a comparação de um produto com um único insumo.

A produtividade está relacionada ao modo como os recursos são utilizados para realizar a produção, sugerindo que o insumo esteja sendo utilizado na quantidade correta e sem excesso. Por outro lado, a eficiência técnica também é uma medida relativa, pois busca relacionar o que foi produzido por unidade de insumo utilizado com o que poderia ser sido produzido com aquele mesmo in- 
sumo. Por suas limitações, apenas as mensurações de produtividade não geram informações suficientes para propiciar melhoria da eficiência (Ferreira \& Gomes, 2009).

Há, na literatura especializada, referências de utilização da DEA para análise da eficiência de organizações privadas, públicas e sem fins lucrativos, como hospitais, entre outras diferentes instituições (Pena, 2008). Dada a complexidade das organizações hospitalares, a mensuração de sua eficiência implica considerar simultaneamente tanto os diversos insumos quanto os produtos resultantes.

O uso da metodologia chamada Análise Envoltória de Dados, do inglês Data Envelopment Analysis (DEA), é uma das possibilidades para calcular a eficiência de diferentes hospitais, permitindo a consideração simultânea de diferentes insumos e produtos (Souza, Scatena, \& Kehrig, 2016).

Essa metodologia pode incorporar múltiplas entradas e saídas, tanto no numerador como no denominador do cálculo da eficiência, não havendo a necessidade da conversão para uma base monetária comum. $A$ medida de eficiência por esse método considera o mix de entradas e saídas, sendo mais abrangente e confiável do que um conjunto de taxas operacionais ou medidores de lucratividade (Fitzsimons \& Fitzsimmons, 2005).

Uma definição importante a ser destacada quando se aplica a DEA refere-se ao termo Unidade Tomadora de Decisão (DMU). As DMU "são organizações produtivas que visam lucro ou beneméritas, como empresas, hospitais, bibliotecas, departamentos de empresas, instituições de ensino etc." (Ferreira \& Gomes, 2009, p. 44). Uma DMU pode ser definida como toda organização que realiza a transformação de um conjunto de entradas (inputs) em um conjunto de saídas (outputs) (Nova, 2002). Segundo essa ótica, uma DMU pode ser um grupo empresarial, uma empresa individual ou uma unidade administrativa.

Os métodos utilizados para estimar a eficiência de unidades produtivas podem ser de dois tipos: paramétrico ou não paramétrico. O paramétrico é o mais tradicional, utilizando regressão múltipla e tendo como função de regressão uma predição probabilística, de modo que as predições representam uma média do desempenho da amostra. Assim, por ter como base os valores médios, a análise produz uma medida imprecisa das meIhores práticas (Pena, 2008).

A DEA é uma metodologia não paramétrica para a mensuração comparativa da eficiência de DMU (Lins, Lobo, Silva, Fiszman, \& Ribeiro, 2007). Isso significa afirmar que essa metodologia não utiliza inferências estatísticas nem se apega a medidas de tendência central, testes de coeficientes ou formalizações de análise de regressão. O método também não exige a existência de relações funcionais entre insumos e produtos (Ferreira \& Gomes, 2009)

Através da DEA, define-se qual a fronteira empírica de produção, que pode ser definida como o maior quantitativo de outputs obtidos com os inputs utilizados em determinado processo produtivo. Desse modo, a DEA permite calcular uma eficiência relativa, derivada das observações realizadas. Os desempenhos das DMU são medidos mediante 


\section{EFICIÊNCIA HOSPITALAR NO SUS: ANÁLISE DE 10 HOSPITAIS DO MIX PÚBLICO-PRIVADO DO ESTADO DE MATO GROSSO}

comparação de seus insumos e resultados com os insumos e resultados de outras DMU inseridas na amostra (Faria, Jannuzzi, \& Silva, 2008). Definidas as DMU com as melhores práticas, a DEA constrói uma fronteira de produção empírica, e o grau de eficiência varia de 0,00 a 1,00 (ou de 0 a 100\%), dependendo da distância da unidade analisada até a fronteira (Lobo, Lins, Silva, \& Fiszman, 2010).

As DMU que estão localizadas na fronteira de produção são denominadas best practices (Simões \& Marques, 2009) ou pares peers, as quais serão o padrão de comparação para as DMU que estão abaixo da fronteira e que lhes são mais semelhantes. A análise realizada pela DEA é chamada de envoltória porque nenhuma DMU pode ficar além da fronteira, ela envolve a todas (Encinas, 2010).

Tanto o modelo como a orientação para input e ou output são os fatores que mais influenciam os resultados obtidos na utilização da DEA (Souza, 2014).

Há dois modelos amplamente utilizados na DEA: o CCR, sigla derivada dos nomes de seus autores (Charnes, Cooper, \& Rhodes, 1978); e o modelo BCC, que é uma extensão do primeiro, e, da mesma forma, se denomina com a sigla advinda dos nomes dos seus autores (Banker, Charnes, \& Cooper, 1984).

No modelo CCR, os retornos constantes de escala (Constant Returns to Scale - CRS) permitem identificar o quanto as organizações procuram maximizar a quantidade combinada dos produtos relacionados à combinação viável dos recursos utilizados (Forgia \& Couttollenc, 2009). Em virtude do retorno constante de escala, espera-se que ocorra uma variação proporcional de produtos a partir da alteração de recursos em todos os níveis de escala. Isso significa dizer que qualquer acréscimo ou redução nos inputs provocará um acréscimo ou redução na mesma proporção nos outputs. Assim, um acréscimo ou redução de $10 \%$ nos inputs causará, em contrapartida, a mesma variação nos outputs (Lobo \& Lins, 2011). Tratando de organizações de saúde, como no caso de hospitais, não é possível estabelecer uma relação assim direta entre inputs e outputs, dada a complexidades dos processos de trabalho considerados e as suas finalidades.

Ao considerar que nem todas as organizações operam com retornos constantes de escala, as medidas de eficiência técnica calculadas pelo modelo CCR podem ser distorcidas pela escala (Forgia \& Couttolenc, 2009). Essa limitação levou ao modelo BCC, que pressupõe a existência dos retornos variáveis de escala (Variable Returns to Scale - VRS).

O modelo CCR não considera os ganhos de escala no cálculo da eficiência, sendo a eficiência relativa da DMU obtida por meio da divisão entre a sua produtividade e a maior produtividade entre as DMU analisadas na observação (Mariano, Almeida, \& Rebellato, 2006). Dessa forma, o modelo CCR permite obter uma estimativa da eficiência total e que pode ser decomposta em eficiência de escala e eficiência técnica interna. Essa última, também denominada eficiência pura, é estimada pelo modelo BCC (Forgia \& Couttolenc, 2009). Ao tratar da definição de eficiência total (CCR), Almeida e Mariano (2006) afirmam que, para seu cálculo, é 
preciso comparar uma DMU com todas as suas concorrentes.

De acordo com Mariano et al. (2006), o modelo BCC compara apenas as DMU que operam em escala semelhante. Assim, a eficiência de uma DMU é obtida dividindo-se sua produtividade pela maior produtividade entre as DMU que apresentam o mesmo tipo de retorno à escala.

Ressalta-se que a diferença entre os dois modelos é que no CCR é considerado best practice, para o estabelecimento da fronteira, apenas uma DMU, ou seja, aquela que, entre todas, apresentar melhores resultados com os insumos disponíveis. Já no BCC, a comparação ocorre entre cada DMU e aquela que apresenta o mesmo tipo de retorno à escala, sendo possível a existência de várias best practices $\mathrm{e}$, consequentemente, várias fronteiras.

A orientação aplicada ao modelo utilizado na DEA é o outro importante fator que influencia os resultados obtidos. Desse modo, o modelo pode ter as seguintes orientações (Bandeira, 2000): orientado a input - utilizado para estimar o nível mínimo possível de uso dos recursos, mantidos os resultados; orientado a output - utilizado para estimar o máximo possível de produtos mantidos fixos os recursos.

O método DEA apresenta-se como uma ferramenta de grande utilidade na avaliação do desempenho de hospitais, tendo o potencial de contribuir na produção de informações que podem auxiliar os gestores em saúde na tomada de decisões visando meIhorias na eficiência dessas organizações.

\section{METODOLOGIA}

Este trabalho caracteriza-se como um estudo multicaso descritivo e exploratório com abordagem quantitativa. A pesquisa pode ser classificada como "estudo de caso", pois, segundo Martins (1994), no estudo de caso, "dedica-se a estudos intensivos do passado, presente e de interações ambientais de uma (ou de algumas) unidade social [...]" (p. 4).

Foram selecionados 10 hospitais distribuídos entre públicos, privados e filantrópicos, os quais estavam localizados em três regiões de saúde do estado de Mato Grosso. Os hospitais selecionados deveriam possuir no mínimo 30 leitos e os privados e filantrópicos, ter pelo menos $50 \%$ dos leitos disponíveis para atendimento SUS. Além disso, foi incluído um hospital recém-inaugurado em função da adoção de um novo modelo de gestão, baseado na transferência da gestão para as Organizações Sociais de Saúde (OSS), que poderia ser comparado com a gestão pública tradicional.

Assim, foram selecionados três hospitais filantrópicos, três privados e quatro públicos. Quanto aos leitos disponíveis para o SUS, também parte desta pesquisa, eles totalizam 822 , ou seja, $88 \%$ do total de leitos dos hospitais selecionados. São 213 leitos (26\%) localizados em hospitais filantrópicos, 139 leitos (17\%) em hospitais privados e 470 leitos $(57 \%)$ em hospitais públicos. Ressalta-se que, por questões éticas, o nome do hospital não será revelado.

Para a aplicação da DEA, inicialmente foi necessário definir o número e quais seriam os itens que comporiam as variáveis inputs e outputs. 


\section{EFICIÊNCIA HOSPITALAR NO SUS: ANÁLISE DE 10 HOSPITAIS DO MIX PÚBLICO-PRIVADO DO ESTADO DE MATO GROSSO}

Em relação à quantidade, respeitou-se o critério reconhecido na literatura: o total de DMU, no mínimo, deve ser igual a duas vezes o total de inputs e outputs para garantir a aplicabilidade da análise de eficiência (Fitzsimmons \& Fitzsimmons, 2005). Assim, considerado o quantitativo de 10 hospitais selecionados para a pesquisa, foi utilizado um número de inputs e outputs restrito ao limite máximo de cinco conjuntos de variáveis.

Foi adotada a classificação de inputs formulada por Marinho e Façanha (2001), consagrada em diversos estudos, que agrupa as variáveis de acordo com três tipos de input: Inputs de trabalho: número de médicos e profissionais de enfermagem (superior, auxiliar e técnico). Para essa variável, o número de profissionais foi ponderado pelo tempo completo equivalente (FTE - Full Time Equivalent), conforme proposto por Ozcan (1995).

Inputs de capital: número de leitos SUS, inclusive de UTI e de isolamento, ao considerar que sua utilização, destacadamente dos leitos de UTI, implica altos custos para um hospital.

Inputs financeiros: valor médio mensal recebido do SUS pelo hospital, referente a internações de 2011 e primeiro semestre de 2012. Tal variável tem sido utilizada na aplicação da DEA, a fim de comparar a assistência hospitalar pública e não pública vinculada ao SUS (Calvo, 2002).

Os dados para a composição dos inputs foram coletados tanto diretamente dos hospitais, mediante visita in $10 \mathrm{co}$, quanto de sistemas de informação em saúde (CNES, SIH-SUS), do Ministério da Saúde, disponibilizados pelo Datasus.

Foram utilizados dois conjuntos de variáveis como outputs: os atendimentos de alta complexidade e um proxy de qualidade.

Internações e Procedimentos de Alta Complexidade (PAC) realizados no período: consideraram-se o número médio mensal de internações SUS e o número médio mensal de procedimentos de alta complexidade realizados para os pacientes internados durante $o$ ano de 2011 e o primeiro semestre de 2012. Esses dados foram obtidos junto ao $\mathrm{SIH} / \mathrm{SUS}$. Essas variáveis coincidem com aquelas utilizadas por Lobo, Silva, Lins, Fiszman, e Bloch (2011), ao analisar a eficiência dos hospitais de ensino no Brasil. Todavia, se diferenciam nesta pesquisa por terem sido somadas as duas variáveis passando a representar um único output, e não separadamente. $O$ "número de procedimentos de alta complexidade" foi utilizado como proxy de case mix, considerado um ajuste fundamental para a validade do modelo DEA (Forgia \& Couttolenc, 2009), ao representar de maneira aproximada a severidade ou complexidade dos casos, além de indicar o uso de um volume maior dos recursos. Essa foi considerada a forma de ajuste mais viável e adequada para esta pesquisa.

Proxy de qualidade: esse é um indicador composto da nota em percentual obtida na verificação dos padrões de conformidade, satisfação dos usuários e pesquisa de condições de trabalho e pelo inverso da taxa de mortalidade hospitalar SUS no ano de 2011 e no primeiro semestre de 2012. Esses itens são oriundos da aplicação anterior do instru- 
mento de avaliação do Programa Nacional de Avaliação de Serviços de Saúde - Pnass (Ministério da Saúde, 2004a). A média desses indicadores forma o proxy (representante) aproximado da qualidade hospitalar. No entanto, quando aplicado para o ano de 2011, foi utilizado apenas o Inverso da Taxa de Mortalidade (ITM) em virtude de os demais itens terem sido coletados no ano de 2012, não se referindo ao período de 2011. $\mathrm{Na}$ avaliação de hospitais universitários, Lobo et al. (2010) sugerem a inserção dessa variável nos outputs, sendo formada pelo score de satisfação dos usuários. Neste estudo, optou-se pela criação de um indicador de qualidade mais completo, visto que os dados obtidos com a aplicação do instrumento do Pnass focam a qualidade em estrutura e processo; já a satisfação dos usuários e 0 inverso da taxa de mortalidade expressam a qualidade com foco nos resultados. Res- salta-se que, em virtude de ser adotada a orientação a output e não se desejar maximizar a taxa de mortalidade, foi utilizado o seu inverso, ou seja, maximizando-se o inverso, minimiza-se a taxa de mortalidade, aspecto importante para a qualidade. O inverso da taxa de mortalidade foi aplicado por Marinho (2001) ao avaliar hospitais do município do Rio de Janeiro. Desse modo, ressalta-se que, neste trabalho, no proxy de qualidade, não se limita a utilizar o score da satisfação dos usuários, mas um indicador mais completo, resultante da avaliação das diversas dimensões propostas pelo Pnass, expressando a qualidade sob a ótica de estrutura, processo e resultado.

De maneira esquemática, o modelo empírico de hospital utilizado na aplicação da DEA pode ser visualizado na Figura 1.

Figura 1. Primeiro modelo empírico de hospital

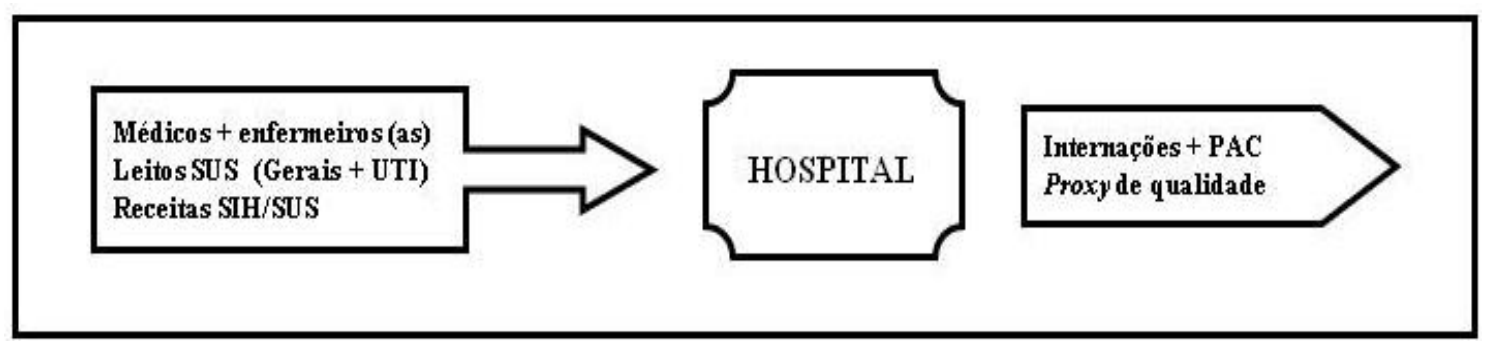

$\mathrm{Na}$ análise dos dados, inicialmente foi feita a correlação de Pearson e elaborada uma matriz de correlação a fim de verificar a relação existente entre as variáveis classificadas como input e output.

Os dados foram processados utilizando 0 software livre SIAD (Sistema Integrado de Apoio à Decisão) e Microsoft Excel e analisados utilizando os dois modelos: inicialmente, foi aplicado o modelo DEA CCR (Constant Returns to Scale - CRS), o qual calcula a eficiência total e pressupõe retornos constantes de escala, sendo possível, assim, verificar os efeitos da escala nos resultados obtidos. Em seguida, foi aplicado o modelo $\mathrm{BCC}$, o qual pressupõe retornos variáveis de escala (VRS - Variable Returns to Scale) e proporciona o cálculo da eficiência técnica, eliminando os efeitos da escala. A orientação aplicada ao modelo DEA foi a output.

Ressalta-se que o modelo empírico de hospital (Figura 1) adotado é o mais comumente 
utilizado na literatura. No entanto, foi inserida uma inovação nesse modelo, que se refere ao componente qualidade na análise DEA conforme sugerido por Lobo et al. (2010) e Marinho e Façanha (2001), que chamam essa variável de output de qualidade. No entanto, tal abordagem ainda não foi encontrada na literatura existente com 0 formato proposto neste trabalho, podendo representar um diferencial nos resultados dessa pesquisa.

A pesquisa que originou este artigo foi submetida ao Comitê de Ética em Pesquisa
(CEP/HUJM), por meio da Plataforma Brasil do Ministério da Saúde, e recebeu parecer favorável em 27 de junho de 2012 (n. 45667).

\section{RESULTADOS E DISCUSSÃO}

Inicialmente, são apresentados os valores dos inputs e outputs para 2011 e primeiro semestre de 2012. Ressalta-se que, no momento da pesquisa, os dados de 2012 ainda não estavam todos disponíveis, mas, como foram utilizados valores médios mensais, os dois períodos foram considerados neste trabalho (Tabela 1).

Tabela 1. Distribuição dos inputs e outputs utilizados para o modelo empírico de hospital adotado, Mato Grosso, 2011 e 2012

\begin{tabular}{|c|c|c|c|c|c|c|c|c|c|c|}
\hline \multirow{3}{*}{ Hospitais } & \multicolumn{6}{|c|}{ Inputs } & \multirow{2}{*}{\multicolumn{2}{|c|}{ Int. + PAC* }} & \multirow{2}{*}{\multicolumn{2}{|c|}{ Proxy }} \\
\hline & \multicolumn{2}{|c|}{ Trabalho } & \multicolumn{2}{|c|}{ Capital } & \multicolumn{2}{|c|}{ Financeiro } & & & & \\
\hline & 2011 & 2012 & 2011 & 2012 & 2011 & 2012 & 2011 & 2012 & 2011 & 2012 \\
\hline Hospital 1 & 368 & 368 & 134 & 172 & $1.332 .560,69$ & $1.232 .300,36$ & 690 & 572 & 96,9 & 62,6 \\
\hline Hospital 2 & 592 & 592 & 280 & 280 & $848.041,46$ & $770.594,20$ & 857 & 725 & 87,9 & 38,6 \\
\hline Hospital 3 & 199 & 199 & 62 & 73 & $82.530,25$ & $134.394,59$ & 149 & 177 & 100 & 73,2 \\
\hline Hospital 4 & 234 & 234 & 85 & 85 & $322.900,74$ & $304.443,08$ & 377 & 374 & 93,3 & 67,0 \\
\hline Hospital 5 & 28 & 28 & 43 & 43 & $34.787,01$ & $23.426,95$ & 62 & 43 & 99,3 & 70,5 \\
\hline Hospital 6 & 309 & 309 & 101 & 101 & $311.496,48$ & $355.386,92$ & 314 & 471 & 96,2 & 68,3 \\
\hline Hospital 7 & 298 & 298 & 46 & 56 & $68.171,22$ & $70.346,30$ & 127 & 132 & 99,8 & 70,7 \\
\hline Hospital 8 & 41 & 41 & 31 & 31 & $51.613,78$ & $53.549,07$ & 109 & 118 & 98,7 & 64,9 \\
\hline Hospital 9 & 90 & 90 & 86 & 86 & $99.209,87$ & $81.956,72$ & 220 & 196 & 98,1 & 57,3 \\
\hline Hospital 10 & 44 & 44 & 63 & 63 & $21.989,19$ & $16.865,79$ & 38 & 29 & 99,6 & 60,1 \\
\hline
\end{tabular}

* PAC - Procedimentos de Alta Complexidade.

Antes de abordar a análise de eficiência para o modelo, apresenta-se a matriz de correlação de Pearson, que evidencia se há correlação entre os itens escolhidos como inputs e outputs e em que grau, de modo semelhante ao aplicado no estudo de Ferreira (2009).

Essa matriz mostra correlação entre os três inputs e outputs considerados na pesquisa, para os dois períodos. Ela foi positiva e forte quando considerados "Internações + PAC" como output, o que já era esperado, visto que os três inputs estão fortemente relacionados ao porte do hospital, ou seja, geralmente, quanto maior o hospital, maior é o número de leitos e funcionários, assim como o faturamento SIH/SUS. Já a correlação foi negativa (e menos forte) quando o proxy de qualidade era o output, informando que, nos hospitais investigados, foi se reduzindo a qualidade à medida que cresciam o porte e a complexidade desses estabelecimentos. 
Paulo Cesar Souza - João Henrique Gurtler Scatena - Ruth Terezinha Kehrig

Tabela 2. Matriz de correlação de Pearson entre inputs e outputs para o modelo empírico de hospital adotado, Mato Grosso, 2011 e 2012

\begin{tabular}{|l|c|c|c|c|c|c|}
\hline \multirow{2}{*}{ Outputs } & \multicolumn{3}{|c|}{ Inputs - 2011 } & \multicolumn{3}{c|}{ Inputs - 2012 } \\
Internações + PAC* & Trabalho & Capital & Financeiro & Trabalho & Capital & Financeiro \\
\cline { 2 - 7 } Proxy de qualidade & 0,88 & 0,91 & 0,90 & 0,90 & 0,90 & 0,86 \\
\cline { 2 - 7 } & $-0,77$ & $-0,89$ & $-0,57$ & $-0,54$ & $-0,81$ & $-0,43$ \\
\hline
\end{tabular}

*PAC - Procedimentos de Alta Complexidade.

A Tabela 3 apresenta o resultado da análise de eficiência para o modelo empírico de hospital adotado, utilizando o software Sistema Integrado de Apoio à Decisão (SIAD), tanto para a eficiência total (CCR) como para a eficiência técnica $(B C R)$, formas distintas de análise do DEA.

Destaca-se que, no cálculo da eficiência to- tal, a produtividade de cada hospital é comparada com o hospital que apresentou a melhor combinação entre recursos e resultados, sendo este chamado de best practice e localizado na fronteira de eficiência, ou seja, compara-se cada hospital com aquele que apresenta as melhores práticas, com o que se torna uma referência (benchmarking) para todos os demais.

Tabela 3. Escores de eficiência total (CCR) e técnica (BCC) de hospitais selecionados, Mato Grosso, 2011 e 2012

\begin{tabular}{|l|c|c|c|c|}
\hline \multirow{2}{*}{ Hospital } & \multicolumn{2}{|c|}{2011} & \multicolumn{2}{c|}{2012} \\
\hline Hospital 1 & Eficiência Total & Eficiência Técnica & Eficiência Total & Eficiência Técnica \\
\cline { 2 - 5 } Hospital 2 & 1,00 & 1,00 & 0,79 & 1,00 \\
\cline { 2 - 5 } Hospital 3 & 0,76 & 1,00 & 0,62 & 1,00 \\
\cline { 2 - 5 } Hospital 4 & 0,83 & 1,00 & 0,63 & 1,00 \\
\cline { 2 - 5 } Hospital 5 & 1,00 & 1,00 & 0,98 & 1,00 \\
\cline { 2 - 5 } Hospital 6 & 1,00 & 1,00 & 1,00 & 1,00 \\
\cline { 2 - 5 } Hospital 7 & 0,75 & 0,99 & 1,00 & 1,00 \\
Hospital 8 & 1,00 & 1,00 & 0,84 & 1,00 \\
\cline { 2 - 5 } Hospital 9 & 1,00 & 1,00 & 1,00 & 1,00 \\
\cline { 2 - 5 } Hospital 10 & 1,00 & 1,00 & 1,00 & 1,00 \\
\hline
\end{tabular}

No ano de 2011, seis hospitais foram considerados eficientes, ou seja, tiveram uma combinação ótima de recursos $(1,00)$, tendo por base aquele de melhor desempenho.

Quanto à natureza jurídica e modelo de gestão, os hospitais 1, 4 e 5 são filantrópicos,
8 e 10 são privados e 9 é público sob gestão direta. Quanto ao porte, se considerados apenas os leitos SUS, dois são hospitais de pequeno porte e quatro, de médio porte (um deles seria classificado como grande porte se considerados os leitos totais). 
No ano de 2012 (incluindo o escore 0,98 pontos do hospital 4), também seis hospitais apresentaram combinação ótima de recursos.

Quanto à natureza jurídica, dois são filantrópicos (4 e 5), dois, públicos (6 e 9), o primeiro sob gestão OSS e o segundo sob gestão direta, e dois, privados (8 e 10). Mantém-se a mesma tipificação de porte, ainda que os hospitais não sejam exatamente os mesmos, nos dois períodos.

Dos hospitais considerados eficientes em ambos os períodos (4, 5, 8, 9 e 10), dois são filantrópicos, um é público sob gestão direta e dois são privados. Dois são de pequeno porte e três, de médio porte.

Há uma observação relevante feita por Marinho e Façanha (2001) a respeito dos resultados obtidos com a DEA utilizando o modelo CCR orientado por output. Para esses autores, o fato de o hospital receber score de eficiência total igual a 1,00 não significa necessariamente um nível irrefutável de eficiência. Isso porque o grau de eficiência é apenas um parâmetro que mensura o consistente equilíbrio entre inputs e outputs. Assim, eficiências máximas não significam ausência de problemas, e também não se recomenda avaliar mal, de imediato, as unidades com scores mais baixos. Nestas, os problemas eventualmente identificados devem suscitar investigações e ajustes em todo o sistema (Marinho \& Façanha, 2001).

De maneira distinta do CCR, para o modelo de eficiência técnica ( $B C C)$, praticamente todos os hospitais investigados mostraram-se eficientes, em ambos os períodos. Como interpretar isso?
O modelo BCC pressupõe que os hospitais investigados são distintos e estão inseridos em contextos diferentes, não sendo possível esperar retorno constante de escala (Ferreira, 2009). Nesse modelo, diferentemente do CCR, a comparação se dá entre os hospitais que operam em escala semelhante, e a eficiência é obtida pela divisão da produtividade de cada hospital pela maior produtividade observada entre o conjunto de hospitais que apresentam o mesmo tipo de retorno à escala (Mariano et al., 2006).

Como foi investigado em número pequeno de hospitais, que também não eram homogêneos, o processo de comparação de hospitais operando em escala semelhante redundou em número ainda menor de hospitais em cada nível de escala. Dessa forma, observou-se aqui um fenômeno já descrito na literatura (Cooper, Seifor, \& Tone, 2000): a menor capacidade de o modelo BCC diferenciar unidades eficientes das menos eficientes quando o número de unidades em comparação é pequeno.

Ressalta-se que ambos os modelos apresentam limitações nos seus resultados.

A eficiência total (CCR) não considera as condições de produção, embora alguns ajustes tenham sido feitos nos dados para melhorar os resultados, por exemplo, a inclusão dos leitos de UTI no total de leitos e a soma dos procedimentos de alta complexidade ao total de internações. Isso teve a finalidade de deixar mais claras as diferenças dos hospitais, relacionadas aos serviços ofertados (Ozcan, 1995) e à complexidade dos atendimentos, medidas que minimizam as limitações do modelo CCR (Forgia \& 
Couttolenc, 2009).

Como já referido, a grande limitação da eficiência técnica $(B C C)$ - a dificuldade em diferenciar níveis distintos de eficiência - deu-se devido ao pequeno número de hospitais operando em escala semelhante. Como os 10 hospitais estudados eram distintos em relação ao porte, à complexidade e ao volume de atendimentos, elementos que determinam a escala de produção, e, além disso, trabalhou-se com o número mínimo de DMU estabelecido pela literatura, a conjunção de tais fatores influenciou o resultado obtido por esse modelo.

Segundo Marinho e Façanha (2001), as diferenças entre os modelos CCR e BCC, principalmente no que se refere ao retorno constante ou retorno variável de escala, não se traduzem em vantagem ou desvantagem de um modelo sobre o outro. A literatura sugere que a questão crucial está na escolha dos elementos que comporão os inputs e outputs.

Assim, mesmo diante das limitações encontradas, os resultados indicam que, quanto à eficiência total (CCR), em 2011, prevaleceram como eficientes os hospitais filantrópicos (três) e privados (dois), com apenas um hospital público entre os seis eficientes. Entre os demais hospitais que se classificaram abaixo da fronteira de eficiência $(1,00) \mathrm{em}$ 2011, os scores mais baixos foram de hospitais públicos $(0,75 ; 0,76)$.

No ano de 2012, o grupo dos eficientes foi mais inclusivo, no que tange à natureza jurídica. Mantiveram-se seis no total, dois filantrópicos, dois privados e dois públicos, entre os quais um sob gestão direta e um sob gestão OSS. Dos hospitais que se situaram abaixo da fronteira de eficiência, scores mais baixos foram encontrados também entre estabelecimentos públicos $(0,62 ; 0,63)$.

Assim, considerando a média do score de eficiência total obtido por cada natureza jurídica, em 2011, destacaram-se os hospitais filantrópicos, todos com score de 1,00, seguidos dos privados $(0,96)$ e dos públicos $(0,84)$. Em 2012, como mostra a Gráfico 1, destacaram-se os privados $(0,95)$, seguidos dos filantrópicos $(0,92)$ e dos públicos $(0,81)$.

Diante disso, conclui-se que, tendo em vista a amostra de hospitais investigados, os modelos utilizados e os inputs e outputs adotados, os hospitais filantrópicos e os privados mostraram-se mais eficientes que os públi$\cos$. Cabe ressalvar novamente que esse resultado não pode ser extrapolado para todos os hospitais do estado de Mato Grosso ou de qualquer região de saúde. 
Gráfico 1. Média do score de eficiência total para os hospitais selecionados, segundo a personalidade jurídica, Mato Grosso, 2011 e 2012

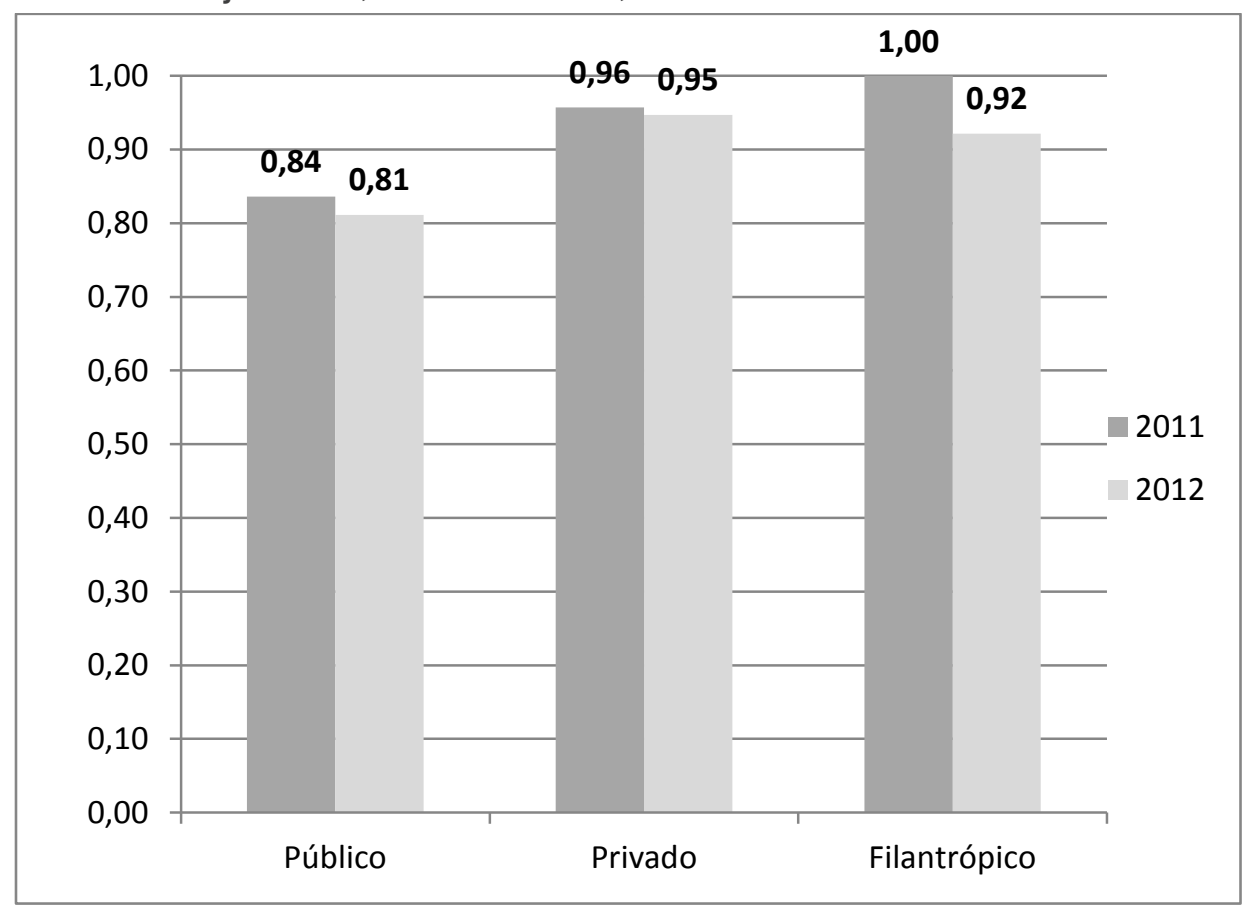

Aprofundando a análise, verificou-se mais uma limitação nos resultados que poderia influenciar a ordem de classificação dos hospitais, espraiando-se também em relação à pontuação obtida por tipo de prestador.

Segundo a literatura, a homogeneidade do universo de estudo, relacionada às variáveis do modelo, é um requisito fundamental, quando da aplicação da DEA (Calvo, 2002; Ferreira, 2009; Ligarda \& Naccha, 2006;
Lobo, 2010; Lobo \& Lins, 2011). Quando o universo de estudo é heterogêneo, recomenda-se a análise de subamostras mais homogêneas (Lobo et al., 2010).

Dois hospitais de estudo ( 1 e 2 ) diferenciam-se bastante dos demais, no que tange ao porte (número de leitos e, consequentemente, de internações) e à complexidade, dada pelos PAC realizados nos dois anos investigados (Tabela 4 ). 
Tabela 4. Características de porte e complexidade dos hospitais selecionados, Mato Grosso, 2011 e 2012

\begin{tabular}{|l|c|c|c|c|c|c|}
\hline Hospitais & \multicolumn{3}{|c|}{$\mathbf{2 0 1 1}$} & \multicolumn{3}{|c|}{$\mathbf{2 0 1 2}$} \\
& Leitos & $\begin{array}{c}\text { Intern. } \\
\text { Hospital 1 }\end{array}$ & PAC & Leitos & Intern. & PAC \\
\hline Hospital 2 & 280 & 589 & 101 & 172 & 473 & 99 \\
\hline Hospital 3 & 62 & 1333 & 24 & 280 & 707 & 18 \\
\hline Hospital 4 & 85 & 377 & - & 85 & 374 & - \\
\hline Hospital 5 & 43 & 61 & 0,8 & 43 & 42 & 1,0 \\
\hline Hospital 6 & 101 & 313 & 1 & 101 & 468 & 3,3 \\
\hline Hospital 7 & 46 & 127 & - & 56 & 132 & - \\
\hline Hospital 8 & 31 & 109 & 0,2 & 31 & 117 & 1,0 \\
\hline Hospital 9 & 86 & 219 & 0,1 & 86 & 196 & - \\
\hline Hospital 10 & 63 & 38 & - & 63 & 29 & - \\
\hline
\end{tabular}

Fonte: elaboração dos autores, com base no CNES e SIH-SUS.

Optou-se, então, pela reprodução da DEA, refazendo os cálculos dos modelos de eficiência total (CCR) e eficiência técnica, agora sem considerar os dois mencionados hospitais, de maior porte e complexidade.

Quanto à eficiência técnica (BCC), o resultado encontrado utilizando apenas oito hospitais foi semelhante ao encontrado no grupo anterior, considerando-se desnecessária sua apresentação. Os resultados do novo cálculo da eficiência total (CCR) são apre- sentados no Gráfico 2.

Os dados mostram que, no ano de 2011, o resultado é semelhante ao encontrado quando foram considerados os 10 hospitais, com a diferença de que, nesse cálculo, o hospital 6 apresenta-se como eficiente, substituindo o hospital 4, que aparecia nessa posição na análise anterior. Interessante notar que o pequeno número de unidades estudadas prejudica o desempenho do modelo DEA, aparecendo muitos hospitais como eficientes. 
Gráfico 2. Eficiência total para os hospitais considerados mais homogêneos, por personalidade jurídica, Mato Grosso, 2011 e 2012

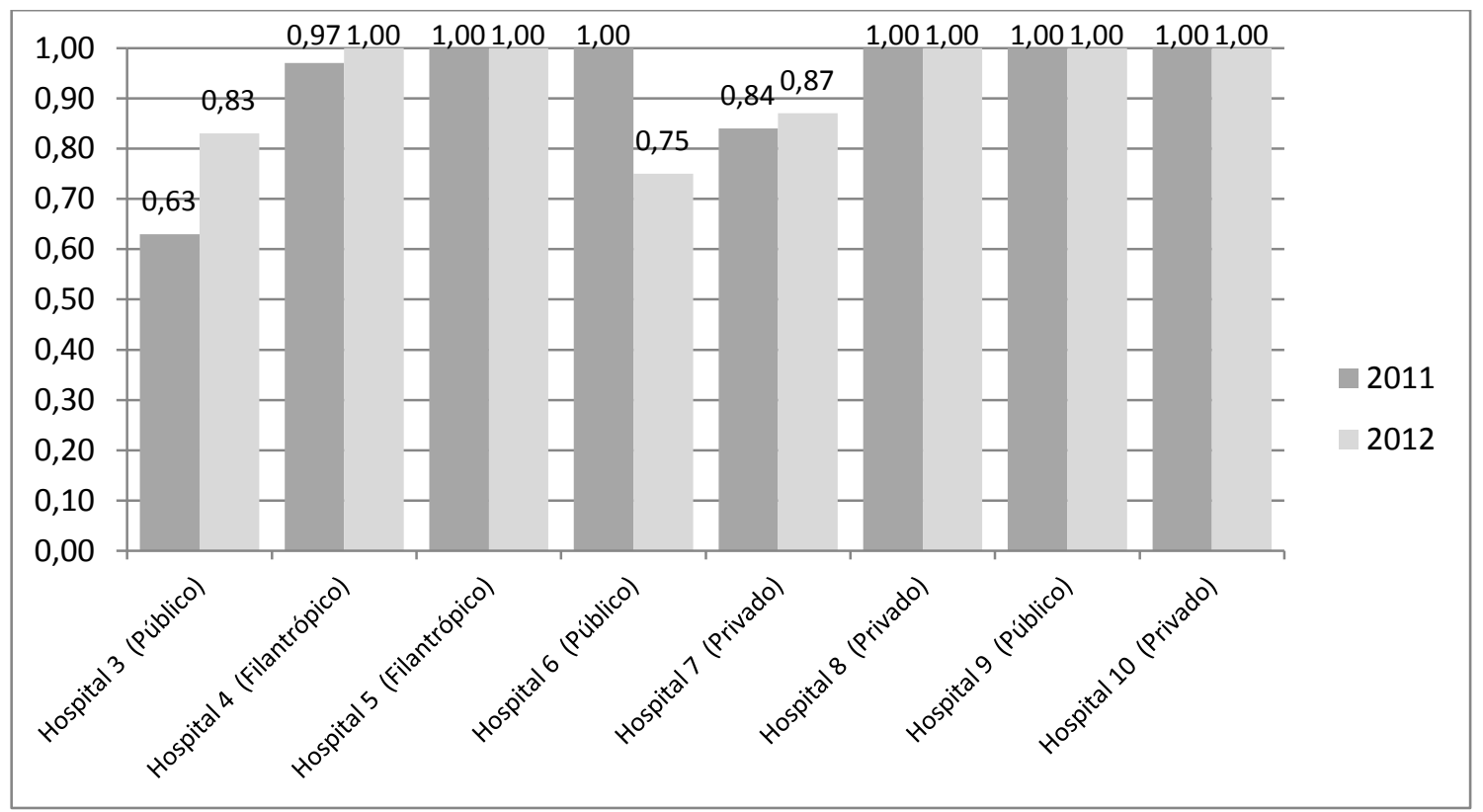

Ressalta-se que o pior resultado nesse ano, com essa forma de análise, foi registrado pelo hospital 3, que é um hospital público sob gestão OSS, ressalvando que esse hospital entrou em funcionamento em 2011, havendo funcionado nesse ano durante apenas quatro meses.

Em 2012, em comparação com a abordagem anterior, que incluía os 10 hospitais, os hospitais considerados eficientes foram quase todos os mesmos, com exceção do hospital 6 , que, nessa nova análise, foi con- siderado ineficiente.

Analisando-se as médias dos scores, por natureza jurídica dos hospitais considerados mais homogêneos, observa-se que filantrópicos prevaleceram sobre os privados e estes, sobre os públicos (Gráfico 3), em ambos os anos. Esse resultado é bastante semelhante ao representado pelo Gráfico 1, com uma variação: no anterior, num dos anos, os privados aparecem em primeiro lugar, seguidos dos filantrópicos. 
Gráfico 3. Média do score de eficiência total para os hospitais considerados mais homogêneos, por personalidade jurídica, Mato Grosso, 2011 e 2012

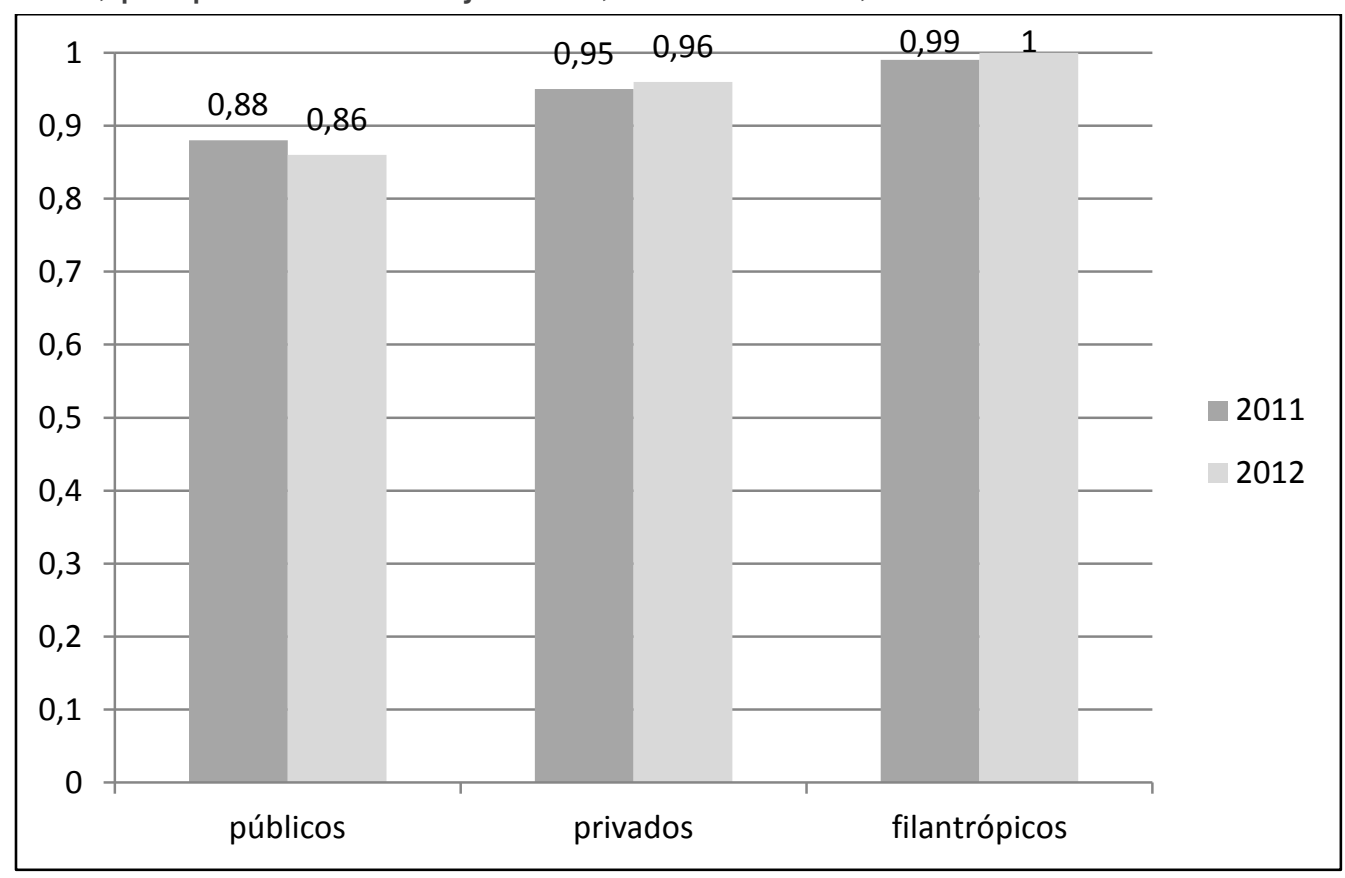

Ressalta-se que esta pesquisa é um estudo de casos múltiplos, no entanto, na DEA, não é possível fugir da comparação, visto que a eficiência calculada é relativa, ou seja, tem como referência o hospital que apresentou o melhor desempenho entre o grupo estudado. Diante disso, o resultado acima tem a limitação de ser oriundo de uma quantidade muito pequena de unidades, limitando o desempenho da DEA.

A fim de qualificar a análise dos resultados encontrados neste estudo, é interessante verificar os resultados de outros estudos similares que se basearam no uso desse método.

Numa dissertação de mestrado, Cesconetto (2006) avaliou a eficiência produtiva de 112 hospitais do SUS de Santa Catarina utilizando dados do SIH/SUS do ano de 2003. Foi aplicado o modelo BCC orientado a output e também a input. Os resultados indicaram que, dos 112 hospitais, apenas 23 estavam na fronteira de eficiência, além de apontar as metas de produção para que cada unidade avaliada alcançasse a eficiência máxima. Entre os 23 eficientes, $61 \%$ eram filantrópicos, $35 \%$ contratados (privados) e $4 \%$ municipais (públicos).

Percebe-se que, embora o foco principal da pesquisa de Cesconetto (2006) não fosse comparar os hospitais públicos, privados e filantrópicos, de modo semelhante a este estudo, em seus resultados, os hospitais filantrópicos destacaram-se com bom nível de eficiência, e os públicos também se apresentam com os menores scores de eficiência.

A fim de verificar se existia diferença no desempenho produtivo dos hospitais públicos e privados do SUS do estado de Mato Grosso que pudesse ser atribuída à natureza administrativa, Calvo (2002) aplicou a DEA por meio do modelo BCC orientado a input 
utilizando dados do SIH/SUS de 1998. Na análise de 80 hospitais, sendo 40 públicos e 40 privados, identificou 12 eficientes entre os públicos e 14 entre os privados. A autora concluiu que não havia diferença entre a proporção de hospitais públicos e privados eficientes, sugerindo que, nos dois tipos de hospitais, a gerência é semelhante quanto à eficiência produtiva.

Percebe-se que o modelo aplicado foi o BCC, que, nesse caso, permitiu diferenciações, em virtude do grande número de hospitais analisados, no entanto a orientação foi input, diferente da orientação adotada neste estudo. Mesmo assim, o resultado encontrado por Calvo (2002) coaduna com o resultado desta pesquisa, que mostrou não haver diferença entre os hospitais quanto à eficiência técnica, ou seja, são todos eficientes tecnicamente.

Dadas as particularidades desta pesquisa, seus resultados e conclusões são circunscritos apenas ao universo de estudo, ainda que se possam destacar semelhanças $e$ diferenças com outros estudos, até mesmo para entender quais delas podem estar relacionadas ao próprio método (número de unidades, modelos, orientação, inputs e outputs etc.) ou ao contexto em que a pesquisa se deu.

\section{CONSIDERAÇÕES FINAIS}

Em que pese número relativamente pequeno de estabelecimentos investigados, a tarefa de realizar a avaliação da eficiência dos hospitais do SUS constituiu-se num grande desafio, sendo o maior deles a coleta de dados primários junto aos hospitais.
Apesar das dificuldades, o objetivo deste trabalho foi alcançado. Verificou-se que os hospitais do SUS, principalmente os públicos, carecem de melhoria na eficiência, visto que, em todas as análises realizadas, os hospitais públicos aparecem com os menores scores de eficiência, estando os filantrópicos sempre mais bem avaliados, seguidos dos privados.

Como se pode visualizar, em ambos os períodos, os públicos apresentaram o menor nível de eficiência total (CCR). Quando eliminados dois hospitais do grupo, por se mostrarem muito distintos dos demais, a média dos scores de eficiência manteve os filantrópicos em primeiro lugar em ambos os períodos, seguidos dos privados, e, por fim, dos públicos. Assim, nesse modelo de análise DEA, no grupo de hospitais pesquisados, os hospitais privados e filantrópicos mostraram-se mais eficientes que os públicos.

\section{REFERÊNCIAS}

Almeida, M. R., \& Mariano, E. B. (2006). A nova administração da produção: Uma sequência de procedimentos pela eficiência. Seminários em Administração. Universidade de São Paulo. São Paulo. Recuperado de http://www.ead.fea.usp.br/ semead/9semead/resultado_semead/an_ indicearea.asp?letra $=B$

Bandeira, D. L. (2000). Análise da eficiência relativa de departamentos acadêmicos: $O$ caso da UFRGS (Dissertação de mestrado, Universidade Federal do Rio Grande do Sul, Porto Alegre, RS).

Banker, R. D., Charnes, A., \& Cooper, W. W. (1984). Some models for estimating techni- 
cal and scale inefficiencies in data envelopment analysis. Management Science, 30(9), 1078-1092. doi:10.1287/mnsc.30.9.1078

Calvo, M. C. M. (2002) Hospitais públicos e privados no Sistema Único de Saúde no Brasil: O mito da eficiência privada no estado de Mato Grosso em 1998 (Tese de doutorado, Universidade Federal de Santa Catarina, Florianópolis, SC).

Cesconetto, A. (2006). Avaliação da eficiência produtiva da rede hospitalar do SUS em Santa Catarina (Dissertação de mestrado, Universidade Federal de Santa Catarina, Florianópolis, SC).

Charnes, A., Cooper, W. W., \& Rhodes, E. (1978). Measuring the efficiency of decision making units. European Journal of Operational Research, 2(6), 429-444. doi:10.1016/0377-2217(78)90138-8

Conselho Nacional de Secretários de Saúde. (2006). SUS: Avanços e desafios. Brasília, DF: Conass.

Cooper, W. W., Seifor, L. M., \& Tone, K. (2000). Data envelopment analysis: A comprehensive text with models, applications, references and DEA - Solver Software. Boston, USA: Kluwer Academic Publishers.

Encinas, R. (2010). Oportunidades de aplicação da análise envoltória de dados em auditorias operacionais do Tribunal de Contas da União (TCC de especialização em orçamento público, Controladoria Geral da União, Brasília, DF).

Faria, F. P., Jannuzzi, P. M., \& Silva, S. J. (2008). Eficiência dos gastos municipais em saúde e educação: Uma investigação através da análise envoltória no estado do Rio de Janeiro. RAP-Revista de Administração Pública, 42(1), 155-177. doi:10.1590/S003476122008000100008.

Ferreira, C. M. C. F., \& Gomes, A. P. (2009). Introdução à análise envoltória de dados: Teoria, modelos e aplicações. Viçosa, MG: Editora UFV.

Ferreira, M. P. (2009). Assistência à saúde nos Departamentos Regionais de Saúde: Um exercício metodológico sobre eficiência e acesso aos serviços de saúde (Tese de doutorado, Faculdade de Medicina, Universidade de São Paulo, São Paulo, SP).

Fitzsimmons, J. A., \& Fitzsimmons, M. J. (2005). Administração de serviços: Operações, estratégia e tecnologia da informação (4a ed.). Porto Alegre, RS: Bookman.

Forgia, G. La, \& Couttolenc, B. F. (2009). Desempenho hospitalar no Brasil: Em busca da excelência. São Paulo, SP: Singular.

Furtado, J. P. (2001). Um método construtivista para a avaliação em saúde. Ciência \& Saúde Coletiva, 6(1), 165-181. doi:10.1590/ S1413-81232001000100014

Ligarda, L., \& Naccha, M. (2006). La eficiencia de las organizaciones de salud a través del análisis envolvente de datos. Microrredes de La Dirección de Salud IV Lima Este 2003. Anales de la Facultad de Medicina, 67(2), 142-151. doi:10.15381/anales.v67i2.1252

Lins, M. P. E., Lobo, M. S. C., Silva, A. C. M., Fiszman, R., \& Ribeiro, V. J. P. (2007). Utilização da análise envoltória de dados (DEA) 
para a avaliação de hospitais universitários brasileiros. Ciência \& Saúde Coletiva, 12(4), 985-998. doi:10.1590/S141381232007000400020

Lobo, M. S. C. (2010). Aplicação da análise envoltória de dados (DEA) para apoio às políticas públicas de saúde: $O$ caso dos hospitais de ensino (Tese de Doutorado, Instituto Alberto Luiz Coimbra de Pós-Graduação e Pesquisa em Engenharia (COPPE), Universidade Federal do Rio de Janeiro, Rio de Janeiro, RJ).

Lobo, M. S. C., \& Lins, M. P. E. (2011). Avaliação da eficiência dos serviços de saúde por meio da análise envoltória de dados. Cadernos de Saúde Coletiva, 19(1), 93-102.

Lobo, M. S. C., Lins, M. P. E., Silva, A. C. M., \& Fiszman, R. (2010). Avaliação de desempenho e integração docente-assistencial nos hospitais universitários. Revista de Saúde Pública, 44(4), 581-590. doi:10.1590/ S0034-89102010000400001

Lobo, M. S. C., Silva, A. C. M., Lins, M. P. E., Fiszman, R., \& Bloch, K. V. (2011). Influência de fatores ambientais na eficiência de hospitais de ensino. Epidemiologia e Serviços de Saúde, 20(1), 37-45. doi:10.5123/ S1679-49742011000100005

Malik, A. M., \& Schiesari, L. M. C. (1998). Qualidade na gestão local de serviços e ações de saúde (Vol. 3). (Série Saúde \& Cidadania). São Paulo, SP: Faculdade de Saúde Pública da Universidade de São Paulo.

Mariano, E. B., Almeida, M. R., \& Rebellato, D. A. N. (2006). Peculiaridade da análise envoltória de dados. XII Simpep, Universi- dade Estadual Paulista, Bauru, SP. Recuperado de http://www.simpep.feb.unesp.br/ anais_simpep_aux.php?e=12

Marinho, A. (2001). Avaliação da eficiência técnica nos serviços de saúde dos municípios do estado do Rio de Janeiro (Texto para discussão, № 842). Brasília, DF: IPEA.

Marinho, A., \& Façanha L. O. (2001). Hospitais universitários: Avaliação comparativa de eficiência técnica (Texto para discussão, № 805). Rio de Janeiro, RJ: IPEA.

Martins, G. A. (1994, 20 semestre). Métodos convencionais e não-convencionais e a pesquisa em administração. Caderno de Pesquisas em Administração, 0(0).

Ministério da Saúde (2004a). Secretaria de Atenção à Saúde. Departamento de Regulação, Avaliação e Controle. Caderno do Programa Nacional de Avaliação dos Serviços de Saúde - PNASS. Brasília, DF.

Ministério da Saúde (2004b). Glossário do Ministério da Saúde: Projeto de terminologia em saúde. Brasília, DF.

Nemes, M. I. B. (2001). Avaliação em saúde: Questões para os programas DST/AIDS no Brasil. Rio de Janeiro, RJ: ABIA.

Nova, S. P. C. C. (2002). Utilização da análise por envoltória de dados (DEA) na análise de demonstrações contábeis (Tese de doutorado, Universidade de São Paulo, São Paulo, SP).

Ozcan, Y. A. (1995). Efficiency of hospital service production in local markets: The balance sheet of U.S. medical armament. 
Socio-Economic Planning Sciences, 29(2), 139-150. doi:10.1016/0038-0121(95)000068

Pena, C. R. (2008). Um modelo de avaliação da eficiência da administraçao pública através do método análise envoltória de dados (DEA). Revista de Administração Contemporânea, 12(1), 83-106. doi:10.1590/S141565552008000100005

Simões, P., \& Marques, C. R. (2009). Avaliação de desempenho dos serviços de resíduos urbanos em Portugal. Engenharia Sanitária e Ambiental, 14(2), 285-294. doi:10.1590/S1413-41522009000200016
Souza, P. C. (2014). Avaliação da qualidade e eficiência da assistência hospitalar em 10 hospitais do SUS no estado de Mato Grosso (Tese de doutorado, Universidade Federal de Mato Grosso, Cuiabá, MT).

Souza, P. C., Scatena, J. H. G., \& Kehrig, R. T. (2016). Aplicação da análise envoltória de dados para avaliar a eficiência de hospitais do SUS em Mato Grosso. Physis Revista de Saúde Coletiva, 26(1), 289-308. doi:10.1590/ S0103-73312016000100016

Tanaka, O. Y., \& Melo, C. (2001). Avaliação de programas de saúde do adolescente: Um modo de fazer. São Paulo, SP: Editora da Universidade de São Paulo. 